\title{
A many-body dissipative particle dynamics with energy conservation study of droplets icing on microstructure surfaces
}

\author{
Chenyang Wang, Xiao Wu, Pengfei Hao, Feng He and Xiwen Zhang ${ }^{*}$ (D)
}

\author{
*Correspondence: zhangxiw@ \\ tsinghua.edu.cn \\ Department of Engineering \\ Mechanics, Tsinghua University, \\ Beijing 100084, China
}

\begin{abstract}
Droplets icing has important applications in real life. The icing process of droplets on microstructure is explored based on the MDPDE method in this study. Firstly, the correctness of the heat transfer model was verified by one-dimensional heat conduction simulation, and then the feasibility of the phase change model was verified by investigating the icing process of droplets. The influence of cold surface temperature, droplet volume and contact angle on freezing time of droplets was discussed, and it was found that the temperature of cold surfaces had a greater effect on freezing. We finally explored the influence of different microstructure surfaces on the icing of droplets, and results showed that the presence of microstructures greatly enhanced the anti-icing effect of the surface. In our research, the contact angle is a relatively large factor affecting the icing of droplets. In addition, it was discovered that the droplet had the strongest ability to delay freezing on the surface of triangle microstructures with a contact angle of $157.1^{\circ}$.

Keywords: MDPDE, Droplet freezing, Microstructure, Anti-icing
\end{abstract}

\section{Introduction}

Icing is a natural phenomenon, which is quite common in life. However, in many fields such as communications and transportation, freezing of liquid droplets will cause a series of consequences. In particular, icing on the surface of aircraft can easily cause flight accidents, endanger personal safety, and cause huge economic losses. The icing problem has attracted investigators' attention in many aspects [1-4], among which there has been plenty of researches on the problem of droplets icing. On the one hand, in the experimental field, Hao P et al. [5] determined the freezing delay time and freezing time of droplets on smooth, micro-structured and micro-nano-structured surfaces. Analysis showed that surface roughness played a vital role in nucleation, and reducing the roughness would increase the free energy barrier of heterogeneous nucleation, leading to significant freezing delay. Then, Jin $\mathrm{Z}$ et al. [6] examined the impact and freezing process of water droplets on different cold cylindrical surfaces. They found that the maximum contact diameter of water droplets in the azimuth direction was generally greater than the maximum contact diameter in the axial direction during the

(c) The Author(s). 2021 Open Access This article is licensed under a Creative Commons Attribution 4.0 International License, which permits use, sharing, adaptation, distribution and reproduction in any medium or format, as long as you give appropriate credit to the original author(s) and the source, provide a link to the Creative Commons licence, and indicate if changes were made. The images or other third party material in this article are included in the article's Creative Commons licence, unless indicated otherwise in a credit line to the material. If material is not included in the article's Creative Commons licence and your intended use is not permitted by statutory regulation or exceeds the permitted use, you will need to obtain permission directly from the copyright holder. To view a copy of this licence, visit http://creativecommons.org/licenses/by/4.0/. 
impact process. Additionally, during the recoil of droplets, the radius of cylindrical surface has a greater influence on the diffusion coefficient of droplet in the circumferential direction, while a smaller influence on the axial diffusion coefficient. Among the freezing process of water droplets, changes in the temperature and radius of cylindrical surfaces did not cause significant influences on the shape of ice droplets. Next, Wang Y et al. [7] observed the freezing of droplets with different contact angles on surfaces of polished silicon wafers and micro-structured silicon wafers, and captured the initiation of ice nucleation of droplets. This investigation found that the free energy barrier of nucleation on graded surfaces was enhanced, which obviously delayed the nucleation of ice. The unique micro-micro-scale topological structure determined the dominant effect of wetting characteristics on ice nucleation. Pan Y et al. [8] studied the effect of surface wettability on the impact of droplets on surface and freezing. The results revealed that droplets spread less on hydrophobic surfaces and the oscillation time is longer. Because droplets contact the surface less, the heat transfer rate is lower, resulting in a delay in icing, and the ice time is significantly prolonged. On inclined surfaces, droplets show the process of sliding and stretching. Because of the increasement of contact area, the total ice time decreases at higher inclination angles. Yutaka Y et al. [9] demonstrated the freezing delay of pre-cooled water droplets on surfaces with four different wetting characteristics, and measured that the freezing delay time enhanced significantly with the increase of surface hydrophobicity. This is because the limited actual contact area between solid and liquid phases limited the possibility of heterogeneous ice nucleation. Reduction of the actual contact area of solid-liquid phase restricts the formation of ice nuclei. When the actual contact area of surface is constant, a surface structure with a smaller radius of curvature is required to further extend the freezing delay time. In addition, Ding B et al. [10] used high-speed cameras to visualize the dynamic behavior of water droplets on superhydrophobic surfaces with different inclination angles and subcooling degrees. It was found that increasing the surface inclination could promote the rebound process of droplets. With gradual decrease of surface temperature, the droplet undergoes complete rebound, partial rebound and no rebound on the surface. The boundary temperatures of three modes are $-31.25^{\circ} \mathrm{C}$ and $-33.75^{\circ} \mathrm{C}$, respectively. Ju J et al. [11] explored the impact and freezing process of hot water droplets on ice surfaces. The water droplet temperature and ice surface temperature were parameterized. Experimental results show that when the temperature of ice surfaces is the same, the increased temperature of water droplets leads to an increase in the maximum diffusion factor and a slight increase in the freezing time. What's more, ice surface temperature has a small effect on the linear contact velocity, and it was also found that the temperature of water droplets has a significant effect on thickness of the thin layer during the expansion process.

On the other hand, in numerical simulation, Hindmarsh JP et al. [12] determined a simple heat balance model. The numerical model established can predict the temperature transition and freezing time of droplets. Subsequently, Chaudhary G et al. [13] conducted a thermal simulation by numerically solving the heat conduction equation based on enthalpy. The numerical calculation results of the droplet freezing are compared with experimental data, and results show that droplets freezing time is in good agreement with experimental data. Then Zhang X et al. [14] proposed a model to predict the icing of droplets. This model assumed that the freezing front was a 
spherical surface and took the effects of gravity and supercooling into account. Simulation results showed that the position of three-phase line, droplet volume and expansion rate were all in great agreement with experimental results. The geometric angle between the gas-liquid interface and horizontal plane tended to decrease most of the time during the solidification process, but suddenly increased at the end of solidification, forming a sharp tip with a sharply curved apex. Zhang X et al. [15] also used the volume of fluid (VOF) multiphase model and the solidification/melting phase transition model to establish a numerical model considering the influence of subcooling on the physical properties of droplet and the influence of dynamic contact angle on the contact line motion to simulate the impact-freezing behavior of droplets. During the impact freezing process of supercooled droplets on a cold hydrophobic surface, three different forms of complete rebound, partial rebound and complete adhesion were found, and a unified rebound and adhesive impact freezing behavior morphology was proposed. Additionally, Bodaghkhani A et al. [16] studied the freezing time of droplets with different wettability in horizontal and oblique directions. Using the twodimensional VOF method, results showed that the lower the surface temperature of droplets, the smaller the static contact angle and the larger the inclination angle, the freezing speed of droplets will be faster.

Despite there having been quantities of studies on droplets icing, there are still unsolved problems. This paper aims to study the icing of droplets on microstructures based on the energy-conserved many-body dissipative dynamics method (MDPDE). Three different microstructure surfaces are proposed, and the icing process of liquid droplets on a flat surface and three types of microstructure surfaces is simulated.

\section{Theoretical description}

\subsection{Nucleation theory}

The nucleation of droplets is affected by many factors and is a complicated process. According to whether nucleation is spontaneous, it can be divided into homogeneous nucleation and heterogeneous nucleation [17-19]. According to classical nucleation theory [20], the nucleation rate J is:

$$
J=K \exp \left(-\frac{\Delta G^{*}}{k_{B} T}\right)
$$

The kinetic parameter $K$ satisfies:

$$
K=K_{0} \exp \left(\Delta \mu / k_{B} T\right)
$$

$K_{0}$ is a constant and is related to the concentration of nucleation sites, $k_{B}$ is the Boltzmann constant, $T$ is the absolute temperature, and $\Delta G^{*}$ is the work required for nucleation, which is expressed as:

$$
\Delta G^{*}=-n^{*} \Delta \mu+\Phi^{*}
$$

Where $n^{\prime \prime}$ is the number of molecules or atoms in the nucleus, $\Phi^{\prime \prime}$ is the effective excess energy on the surface of nucleus, and $\Delta \mu$ is the chemical potential difference between the liquid phase and crystalline phase, which is expressed as [21]:

$$
\Delta \mu=k_{B} T \ln \left(P_{s w} / P_{s i}\right)
$$

$$
P_{s w} \text { and } P_{s i} \text { are the saturated vapor pressures of water and ice, respectively. }
$$


In the case of non-uniform nucleation, the energy barrier for nucleation is lower than that of uniform nucleation. Therefore, the nucleation efficiency parameter $\phi$ is introduced to describe the influence of inhomogeneity [22]:

$$
\phi=\frac{(2+\cos \theta)(1-\cos \theta)^{2}}{4}
$$

Where $\theta$ is the contact angle between the ice and the substrate. Relative to homogeneous nucleation, heterogeneous nucleation requires a lower energy barrier. In most cases, droplet icing starts under heterogeneous nucleation.

\subsection{Numerical method}

MDPDE is a particle-based numerical method. Because of its ascendency in capturing micron-level mesoscopic features such as droplet splash during droplet collisions, this method has great advantages in simulating droplet collisions. So far, there have been quantities of related studies [23-28]. Many-body dissipative dynamics method (MDPD) is based on the original dissipative dynamics method (DPD) [29, 30], and by modifying the original conservative force term that only contains repulsive force into a multi-body potential energy function, it solves the problem that a large number of gas-phase particles are required for the traditional DPD method to simulate gas-liquid two-phase flow. The temperature of the original DPD method is constant and there is no heat transfer. After adding an energy equation to the original equation of state to calculate the internal energy of each particle, the temperature simulation is achieved, and the energyconserved dissipative dynamics method (DPDE) is obtained. Combining the above two models, the MDPDE method is obtained, and the critical theoretical formulae are as follows:

$$
\begin{aligned}
& F_{i j}^{C}=A \omega_{c}\left(r_{i j}\right) \overrightarrow{e_{i j}}+B\left(\overline{\rho_{i}}+\overline{\rho_{j}}\right) \omega_{d}\left(r_{i j}\right) \overrightarrow{e_{i j}} \\
& F_{i j}^{D}=-\gamma \omega_{D}\left(r_{i j}\right)\left(\overrightarrow{e_{i j}} \cdot \overrightarrow{v_{i j}}\right) \overrightarrow{e_{i j}} \\
& F_{i j}^{R}=\xi \omega_{R}\left(r_{i j}\right) \theta_{\xi}(\Delta T)^{-1 / 2} \overrightarrow{e_{i j}}
\end{aligned}
$$

Where $F_{i j}^{C}, F_{i j}^{D}$ and $F_{i j}^{R}$ are the conservative force, dissipative force and random force of the system respectively. $A$ and $B$ are the attraction and repulsion coefficients between particles, $\omega_{c}\left(r_{i j}\right)$ and $\omega_{d}\left(r_{i j}\right)$ are their respective weight functions. Also, $\gamma$ and $\xi$ are the coefficients of dissipation force and random force, respectively, and they satisfy the thermodynamic fluctuation-dissipation theorem. $\theta_{\xi}$ is a white noise term with zero mean unit variance.

The local density function is:

$$
\overline{\rho_{i}}=\sum_{i \neq j} 15 /\left(2 \pi r_{d}^{3}\right)\left(1-r_{i j} / r_{d}\right)^{2}
$$

For solid and fluid particles, according to the study of Wang L et al. [25], the solidliquid interaction function is: 
Table 1 MDPDE related parameters

\begin{tabular}{lll}
\hline name & symbol & value \\
\hline particle number density & $\rho_{l \prime}, \rho_{s}$ & $8.6,7.9$ \\
Attractive coefficient & $A_{l,} A_{s}$ & $-200,-181.8$ \\
Repulsive coefficient & $B_{l \prime}, B_{s}$ & $25.0,27.5$ \\
Dissipation coefficient & $\gamma_{l}, \gamma_{s}$ & $2.0,10.0$ \\
Cut-off range & $r_{c}$ & $1.0 I_{R}$ \\
Boltzmann temperature & $k_{B} T$ & $1.0 T_{R}$ \\
Heat capacity & $C_{V}^{\prime}, C_{V}^{s}$ & $3.5 \mathrm{e} 16,1.6 \mathrm{e} 16$ \\
Equilibrium temperature & $T_{e}$ & $0.91 T_{R}$ \\
Latent heat & $L$ & 0.27 \\
Time step & $\Delta t$ & $0.01 t_{R}$ \\
Length unit & $I_{R}$ & $1 \mathrm{e}-4 \mathrm{~m}$ \\
Time unit & $t_{R}$ & $0.1 \mathrm{~s}$ \\
Mass unit & $M_{R}$ & $1.2 \mathrm{e}-8 \mathrm{~kg}$ \\
Energy unit & $E_{R}$ & $4.14 \mathrm{e}-21 \mathrm{~J}$ \\
Temperature unit & $T_{R}$ & $300 \mathrm{~K}$ \\
\hline
\end{tabular}

$$
F_{s l}^{C}=\left\{\begin{array}{c}
F b\left(1-r_{i j} / r_{b}\right)\left(r_{i j} / r_{b}\right) ;\left(r \leq r_{b}\right) \\
-F a\left(r_{b}-r_{i j}\right)\left(r_{b}-r_{a}\right) ;\left(r_{b} \leq r \leq r_{a}\right) \\
-F a\left(r_{s l}-r_{i j}\right)\left(r_{s l}-r_{a}\right) ;\left(r_{a}<r \leq r_{s l}\right)
\end{array}\right.
$$

Energy-related parts:

$$
\begin{aligned}
& C_{v} \frac{d T_{i}}{d t}=\sum_{j \neq i}\left(q_{i j}^{V_{h}}+q_{i j}^{C_{h}}+q_{i j}^{R_{h}}\right) \\
& q_{i j}^{V_{h}}=\frac{1}{2 C_{v}}\left\{\omega^{D}\left(r_{i j}\right)\left[\gamma_{i j}\left(\overrightarrow{e_{i j}} \cdot \overrightarrow{v_{i j}}\right)^{2}-\xi_{i j}^{2} / m_{i}\right]-\xi_{i j} \omega^{R}\left(r_{i j}\right)\left(\overrightarrow{e_{i j}} \cdot \overrightarrow{v_{i j}}\right) \theta_{\xi}\right\} \\
& q_{i j}^{C_{h}}=\kappa_{i j} \omega^{H C}\left(r_{i j}\right)\left(1 / T_{i}-1 / T_{j}\right) \\
& q_{i j}^{R_{h}}=\alpha_{i j} \omega^{H R}\left(r_{i j}\right) \xi_{i j}^{e} \Delta t^{-1 / 2}
\end{aligned}
$$

Where $C_{v}$ is the specific heat capacity, $q_{i j}^{V_{h}}, q_{i j}^{C_{h}}$ and $q_{i j}^{R_{h}}$ are the internal energy change items caused by viscosity, collision and random thermal motion, respectively. Among them:

$$
\alpha_{i j}=\sqrt{2 k_{B} \kappa_{i j}}
$$
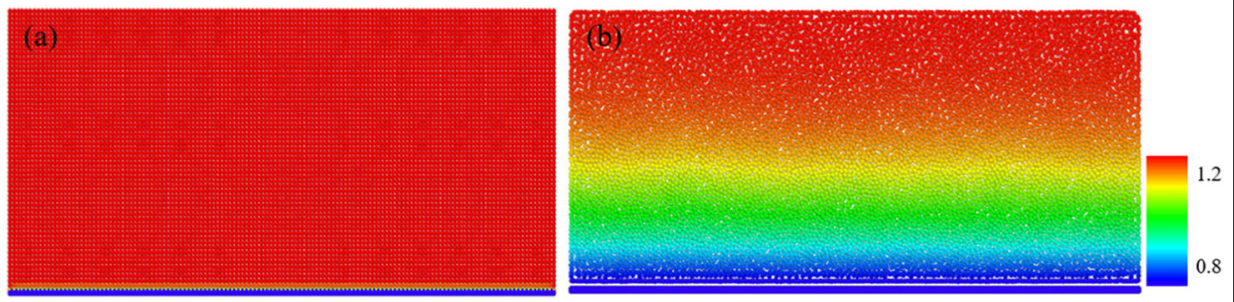

Fig. 1 (a) One-dimensional heat conduction calculation domain (b) $t=20$ temperature distribution 


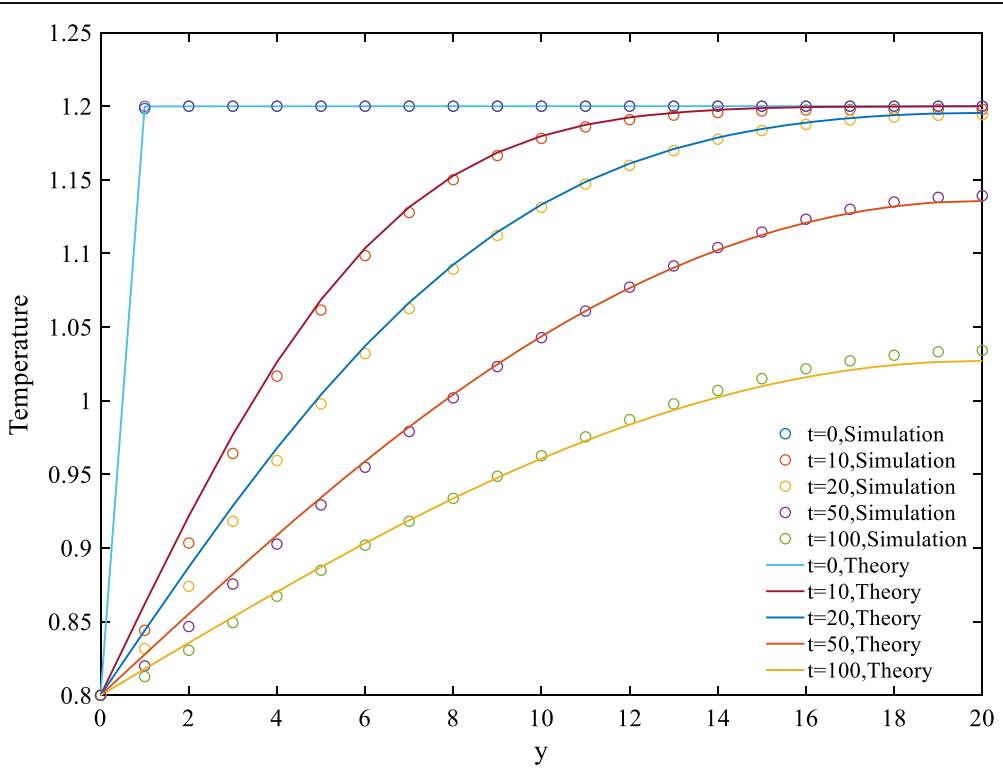

Fig. 2 Temperature in the $y$ direction changes with time in the calculation domain

$$
\begin{aligned}
& \omega^{D}\left(r_{i j}\right)=\left\{\begin{array}{cc}
\left(1-\frac{r_{i j}}{r_{c}}\right)^{S_{v}} ; & r_{i j}<r_{C} \\
0 ; & r_{i j} \geq r_{C}
\end{array}\right. \\
& S_{v}=C_{1}+C_{2}\left(T^{2}-1\right)
\end{aligned}
$$

When phase change occurs, the consumption of latent heat needs to be considered. The relationship between temperature, latent heat and internal energy is as follows:

$$
T=\left\{\begin{array}{cc}
(\varepsilon-L) / C_{v} ; & \varepsilon>C_{v} T_{m}+L \\
T_{m} ; & C_{v} T_{m}+L \geq \varepsilon \geq C_{v} T_{m} \\
\varepsilon / C_{v} ; & C_{v} T_{m}>\varepsilon
\end{array}\right.
$$

Where $L$ and $\varepsilon$ represent latent heat and internal energy, respectively.

\section{Results and discussion}

\subsection{Verification example one: one-dimensional heat conduction problem}

Based on the above numerical model, the one-dimensional heat conduction problem was first verified. Relevant simulation parameters are shown in Table 1, and the symbol with $l$ represents liquid phase, while $s$ represents solid phase. As shown in Fig. 1a, a rectangular two-dimensional computational domain is established. Periodic boundary

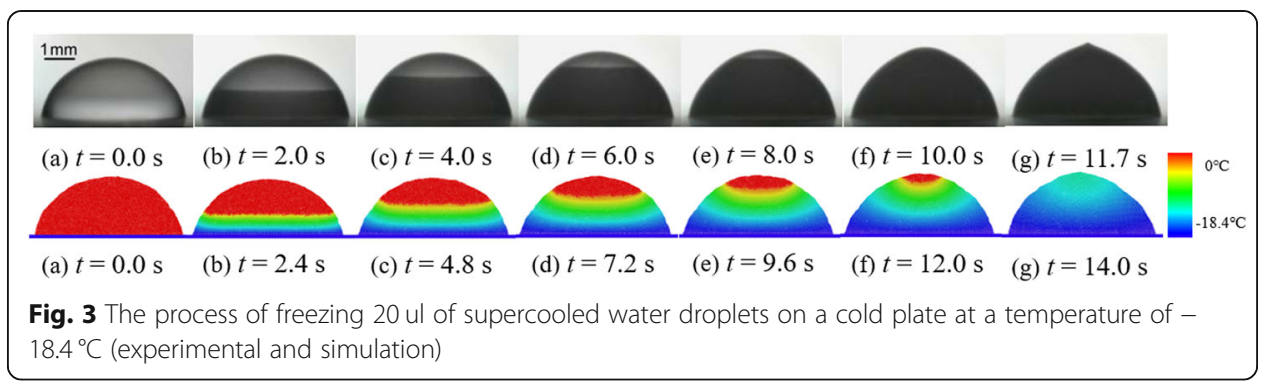




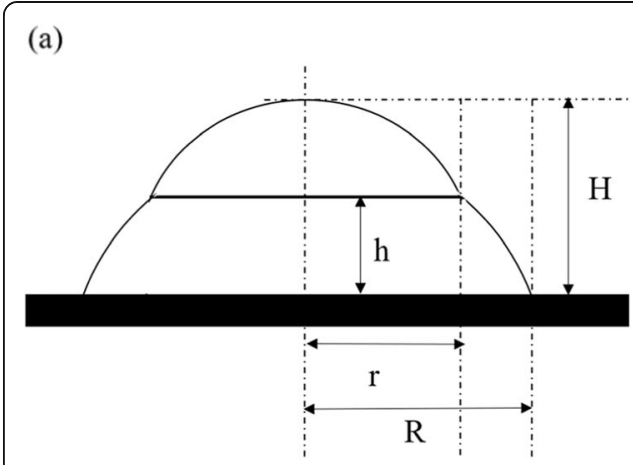

(b)

Fig. 4 (a) Freezing stage (b) $R^{*}$ and $H^{*}$ of droplet change with $t^{*}$

condition is used in the $x$-direction, and the lower boundary in the $y$-direction fixed temperature is $0.8 T_{R}$. Above the solid wall is a fluid area composed of liquid particles with a number density of 8.6 and temperature of $1.2 T_{R}$. 7980 fluid particles are consumed and the total number of solid wall particles is 1353 . Figure $1 \mathrm{~b}$ is the temperature distribution in the calculation domain when $t=10 t_{R}$. It can be seen that as time progresses, the temperature of the cold wall gradually spreads to the liquid.

Figure 2 shows the temperature change with time in the $y$ direction of the calculation domain. It can be seen that the numerical solution obtained based on the MDPDE model is in great consistency with the theoretical value. We count the errors of different time steps and find that they are all within the range of $1 \%$, which verifies the correctness of the heat transfer model.

\subsection{Verification example two: the droplets on the cold wall freeze}

The initial temperature of droplets is $0^{\circ} \mathrm{C}$ in all simulation situations in our study. When the droplet is placed on a cold surface, it begins to nucleate when its highest temperature drops to $T_{n}$, and then the entire droplet begins to condense.

Next, the icing process of droplets was simulated and compared with the experimental results of Zhang X et al. [31]. Results are shown in Fig. 3, and the same label of experiment and simulation corresponds to the same proportion of entire icing time. The size of droplets in our simulation is $20 \mathrm{ul}$, the temperature of cold wall surfaces is maintained at $-18.4{ }^{\circ} \mathrm{C}$, and the contact angle of the droplet is $78^{\circ}$. In the experiment, the droplet experienced $11.7 \mathrm{~s}$ from the end of recalescence to complete freezing. The simulation result was $14 \mathrm{~s}$, and the error (19.7\%) between the two was within a reasonable range. Corresponding experimental and simulated icing processes are shown in Fig. 3 . The sharp angle obtained by simulation is $148^{\circ}$, and the experiment is $140^{\circ}$. The error between the two is only $5.7 \%$, which verifies the accuracy of the numerical results.

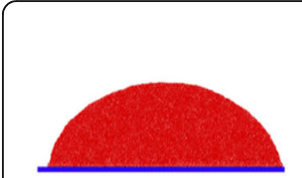

(a)

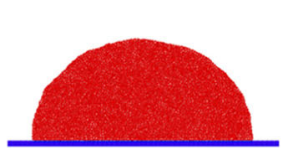

(b)

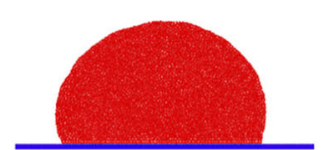

(c)

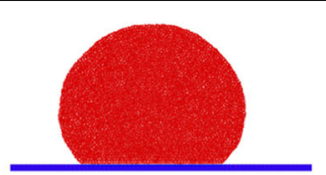

(d)

Fig. 5 The contact angles are $(\mathbf{a}) \theta=78^{\circ}$ (b) $\theta=90^{\circ}$ (c) $\theta=110^{\circ}$ (d) $\theta=130^{\circ}$ 
Table 2 Simulation related data

\begin{tabular}{llllll}
\hline Droplet volume $(\mathbf{u})$ & $\mathbf{1 0}$ & & $\mathbf{2 0}$ & $\mathbf{1 3 0}$ \\
\cline { 2 - 5 } Contact angle $\left({ }^{\circ}\right)$ & $\mathbf{7 8}$ & $\mathbf{9 0}$ & & $\mathbf{1 1 0}$ & -20 \\
\hline Cold surface temperature $\left({ }^{\circ} \mathrm{C}\right)$ & -5 & -10 & -15 & -15
\end{tabular}

Figure $4 \mathrm{a}$ is a moment of the icing process, and Fig. $4 \mathrm{~b}$ is the normalized result of height $H *(h / H)$ and radius $R *(r / R)$ of corresponding icing fronts during the icing process of the droplet. It can be seen that the results of simulations and experiments are relatively approximate, which verifies the correctness of the icing model again.

\subsection{Icing on a flat surface}

Based on the above verification results, the feasibility of the MDPDE method to simulate the icing process of droplets is proved. In this study, by means of changing the volume, contact angle of droplets and temperature of solid walls, the change of freezing time of droplets with them was explored. The icing process of droplets starts from the end of recalescence to complete freezing. The specific values of the droplet volume, contact angle and wall temperature are shown in Fig. 5 and Table 2.

Figure 6 shows the result of freezing time of 10ul droplets with distinct contact angles on cold surfaces with different temperatures. It can be proved that the freezing time enhances with the increase of the droplet contact angle and solid wall temperature. When the solid wall temperature is higher, the variation in freezing time between droplets with diverse contact angles is more apparent. From Fig. 6a and b, it can be seen that under identical conditions, the larger the volume of droplets, the longer the corresponding freezing time. In the case of an equivalent contact angle, temperature of different cold wall makes a great divergence in freezing time. In summary, the contact angle has a relatively significant influence on droplets freezing time.

\subsection{Freezing on the surface with microstructures}

Eventually, the icing of droplets on surfaces with microstructures is studied. As shown in Fig. 7, three different types of microstructures are considered, namely square,
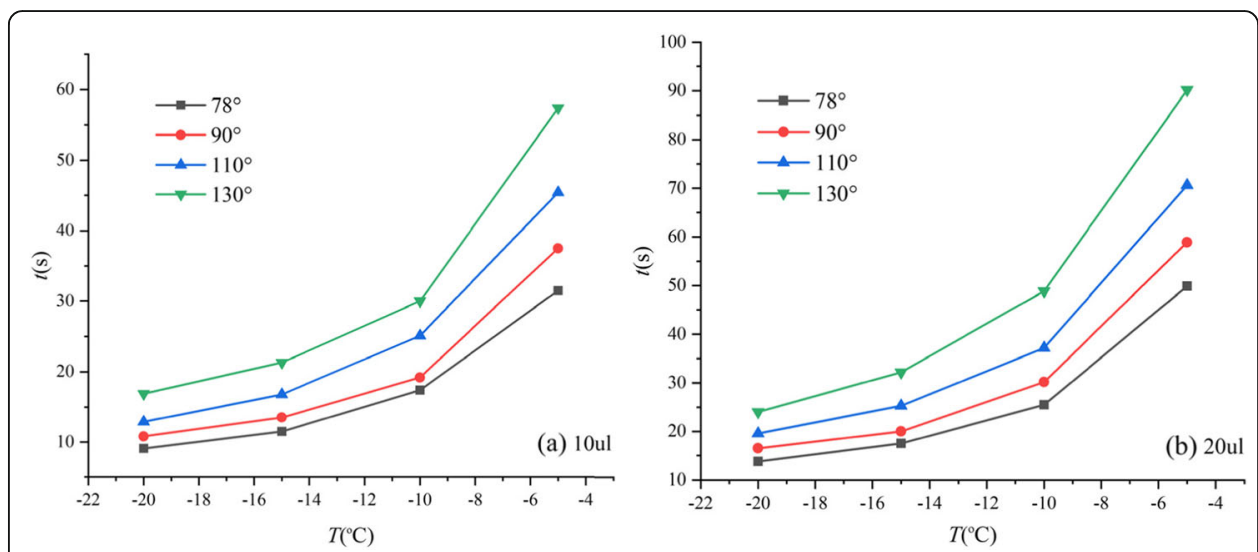

Fig. 6 Freezing time of (a) 10ul (b) 20ul droplets on cold surfaces with different contact angles varies with wall temperature 


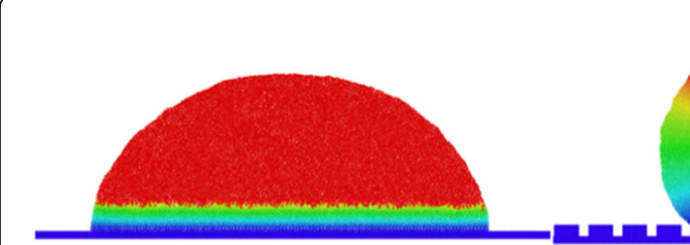

(a)

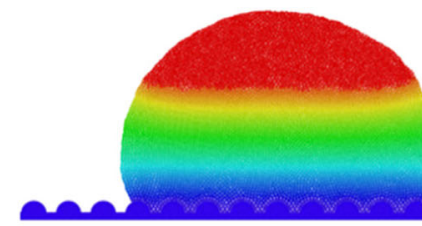

(c)

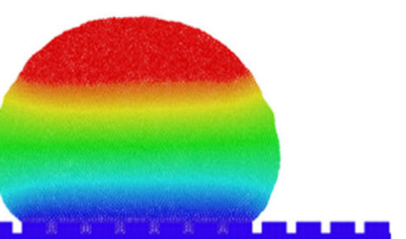

(b)

Fig. 7 Freezing of droplets on surfaces of different microstructures. (a) plane (b) square (c) semicircle (d) triangle

semicircle and triangular. The height and bottom width of these three patterns are kept identical respectively. Droplet volume in this simulation includes two cases: $20 \mathrm{ul}$ and 40ul. The temperature of cold wall surfaces is kept at $-15.9^{\circ} \mathrm{C}$. The icing process of droplets starts from the end of recalescence to the end of droplets' complete freezing.

Figure 8 is the change of freezing time of droplets on different cold surfaces with parameter $A$ (the force between solid and liquid). Contact angles of droplets on plane and three types of microstructure surfaces corresponding to different parameter $A$ are shown in Tables 3 and 4 , and the maximum $\theta$ corresponding to each $A$ is bolded. It can be seen that with the increase of the plane contact angle, the freezing time on four cold surfaces all improves, and the increase of droplet volume will lead to the enhancement of freezing time. It can be concluded from Fig. 8 that the presence of microstructures greatly enhances the anti-icing effect of a surface. For each solid-liquid force $A$, a maximum freezing time exists. According to the results, surfaces with the largest contact angle correspond to the longest freezing time in each case, which means the foremost determinant of freezing time of different microstructure surfaces is the contact
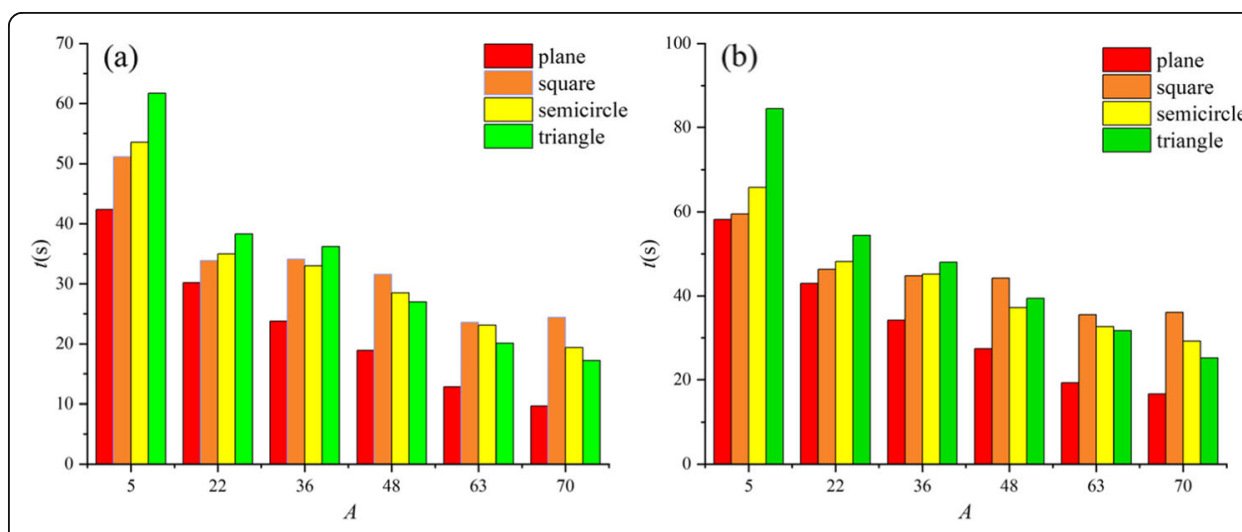

Fig. 8 Freezing time of (a) $20 u$ (b) $40 u$ d droplets on surfaces of different microstructures 
Table 3 Contact angles of 20ul droplets on surfaces of different microstructures

\begin{tabular}{|c|c|c|c|c|}
\hline$A$ & plane & square & semicircle & triangle \\
\hline 5 & $150.4^{\circ}$ & $150.2^{\circ}$ & $150.9^{\circ}$ & $157.1^{\circ}$ \\
\hline 22 & $125.5^{\circ}$ & $129.6^{\circ}$ & $133.6^{\circ}$ & $135.1^{\circ}$ \\
\hline 36 & $103.8^{\circ}$ & $127.4^{\circ}$ & $130.5^{\circ}$ & $134.9^{\circ}$ \\
\hline 48 & $83.1^{\circ}$ & $126.7^{\circ}$ & $116.9^{\circ}$ & $110.5^{\circ}$ \\
\hline 63 & $63.1^{\circ}$ & $114.6^{\circ}$ & $103.8^{\circ}$ & $86.3^{\circ}$ \\
\hline 70 & $40.1^{\circ}$ & $102.6^{\circ}$ & $80.1^{\circ}$ & $73.6^{\circ}$ \\
\hline
\end{tabular}

angle. In general, for a fixed solid-liquid force $A$, the larger the contact angle, the better the corresponding delayed icing effect, which is consistent with the experimental results of Liu Y et al. [32].

Taking the icing process of 40ul droplets on four types of surfaces under solid-liquid force $A=48$ as an example, the temperature distribution inside the droplets during the icing process is shown. It can be seen from Fig. 9 that after adding the microstructure, the heat transfer between the droplet and the wall becomes slower, and the droplet on the plane completely solidifies into an ice phase at $18.9 \mathrm{~s}$. On the other three types of microstructures, the liquid droplets were completely solidified in about $30 \mathrm{~s}$. Next, further analysis of the results in each case is carried out.

Count the longest freezing time among the four surfaces corresponding to each fixed solid-liquid force $A$, and the result is shown in Fig. 10, where 1-6 on the abscissa correspond to contact angles $\theta=157.1^{\circ}, 135.1^{\circ}, 134.9^{\circ}, 126.7^{\circ}, 114.6^{\circ}, 102.6^{\circ}(20 \mathrm{ul}), \theta=$ $144.1^{\circ}, 133.6^{\circ}, 124.0^{\circ}, 118.2^{\circ}, 99.8^{\circ}, 98.5^{\circ}(40 \mathrm{ul})$, respectively. Obviously, it can be known that under the premise of a single variable, either the contact angle or volume of droplets increases, the freezing time improves significantly. The greater the hydrophobicity, the more beneficial it is to prevent freezing. In addition, the droplet has the strongest ability to delay icing on triangular microstructure surfaces with a contact angle of $157.1^{\circ}$.

\section{Conclusion}

This paper explored the influence factors of droplets icing on a flat surface and the effect of the type of micro-structured surface on droplets icing. The results demonstrated the following:

Table 4 Contact angles of $40 u$ droplets on surfaces of different microstructures

\begin{tabular}{|c|c|c|c|c|}
\hline$A$ & plane & square & semicircle & triangle \\
\hline 5 & $142.1^{\circ}$ & $141.9^{\circ}$ & $142.2^{\circ}$ & $144.1^{\circ}$ \\
\hline 22 & $119.2^{\circ}$ & $122.0^{\circ}$ & $124.9^{\circ}$ & $133.6^{\circ}$ \\
\hline 36 & $99.0^{\circ}$ & $119.1^{\circ}$ & $120.7^{\circ}$ & $124.0^{\circ}$ \\
\hline 48 & $79.3^{\circ}$ & $118.2^{\circ}$ & $104.4^{\circ}$ & $102.2^{\circ}$ \\
\hline 63 & $51.5^{\circ}$ & $99.8^{\circ}$ & $90.7^{\circ}$ & $85.8^{\circ}$ \\
\hline 70 & $43.8^{\circ}$ & $98.5^{\circ}$ & $80.3^{\circ}$ & $67.9^{\circ}$ \\
\hline
\end{tabular}




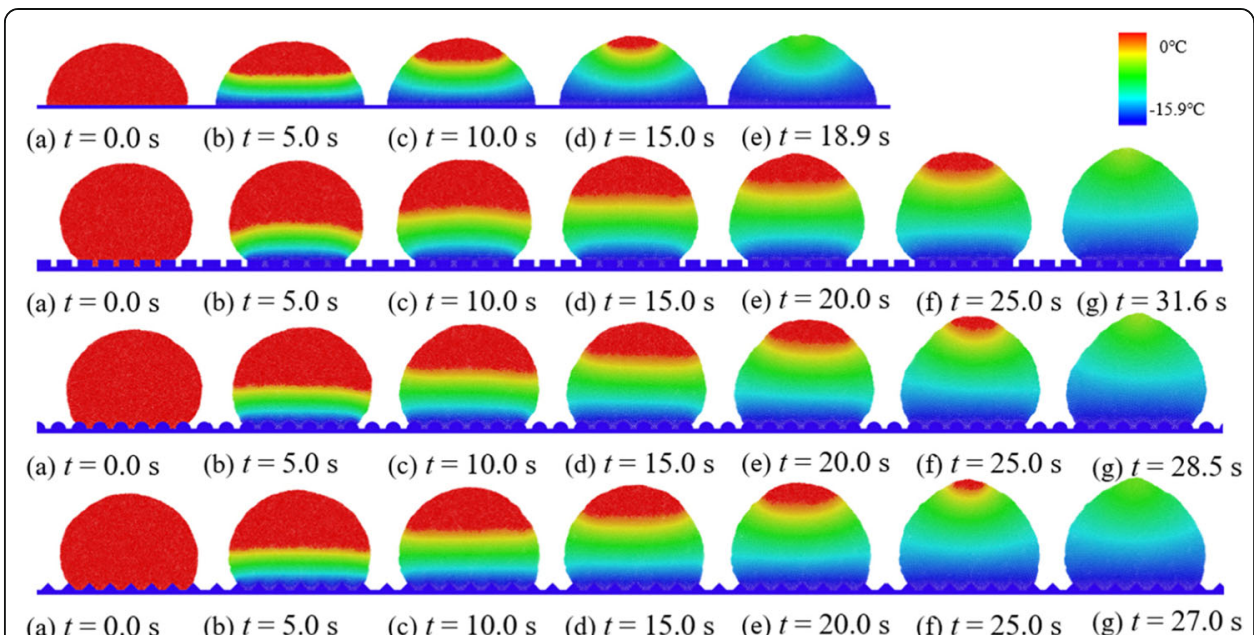

Fig. 9 Temperature distribution of $40 u$ droplets when freezing on different surfaces

(1) The freezing time enhances with the increase of the droplet contact angle, droplet volume and solid wall temperature. The contact angle has a relatively significant influence on droplets freezing time;

(2) The presence of microstructures obviously improves the anti-icing effect of a surface. The droplets freezing time of different microstructures mainly depends on the contact angle. Freezing time of the same surface increases with enhancement of the contact angle. Under the same solid-liquid force $A$, the contact angle of droplets on a microstructure surface is larger, so its effect of delaying freezing is better than the flat surface;

(3) The droplet has the strongest capability to delay icing on triangle microstructure surfaces with a contact angle of $157 \cdot 1^{\circ}$.

Based on the research in this paper, the hydrophobicity of a surface should be ensured as much as possible, and the ice-repellency of a cold surface can be increased by

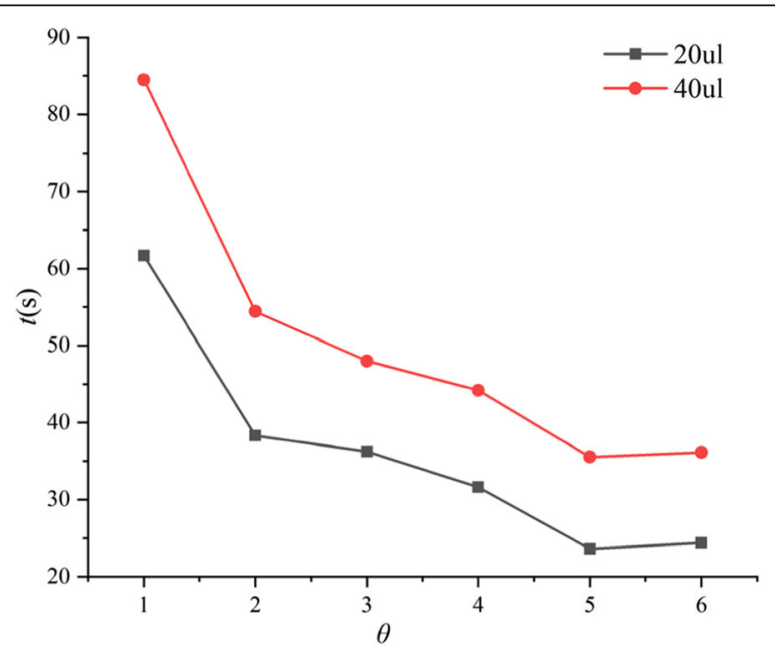

Fig. 10 The maximum freezing time changes with different contact angles 
adding microstructures on the plane. This research has crucial guiding significance for actual anti-icing problems.

\section{Acknowledgements}

The authors gratefully acknowledge the financial support of the National Numerical Windtunnel Project (Grant Nos. NNW2019ZT2-B05, NNW2019ZT2-B26), and the National Natural Science Foundation of China (Grant Nos.11972215, 12072174)

\section{Authors' contributions}

CW finished the numerical simulations, and XW performed post-processing on the results. All authors analyzed the data and were involved in writing the manuscript. All authors read and approved the final manuscript.

\section{Funding}

This work was supported by the National Numerical Windtunnel Project (Grant Nos. NNW2019ZT2-B05, NNW2019ZT2B26), and the National Natural Science Foundation of China (Grant Nos.11972215, 12072174).

\section{Availability of data and materials}

All the data and materials are available in this paper.

\section{Declarations}

\section{Competing interests}

The authors do not have any possible conflicts of interest.

Received: 14 May 2021 Accepted: 15 July 2021

Published online: 04 November 2021

\section{References}

1. Golovin K, Kobaku SPR, Lee DH, DiLoreto ET, Mabry JM, Tuteja A (2016) Designing durable icephobic surfaces. Sci Adv 2(3):1501496. https://doi.org/10.1126/sciadv.1501496

2. Shen J, Lu H, Li R, Chen X, Ma H (2020) The thermochemical non-equilibrium scale effects of the high enthalpy nozzle. Adv Aerodyn 2:20. https://doi.org/10.1186/s42774-020-00044-9

3. Li R, Xu W, Zhang D (2021) Impacts of thermal and mechanical cycles on electro-thermal anti-icing system of CFRP laminates embedding sprayable metal film. Materials 14(7):1589. https://doi.org/10.3390/ma14071589

4. Morelli M, Bellosta T, Guardone A (2021) Efficient radial basis function mesh deformation methods for aircraft icing. J Comput Appl Math 392:113492. https://doi.org/10.1016/j.cam.2021.113492

5. Hao P, LV C, Zhang X (2014) Freezing of sessile water droplets on surfaces with various roughness and wettability. Appl Phys Lett 104(16):161609. https://doi.org/10.1063/1.4873345

6. Jin Z, Zhang H, Yang Z (2017) The impact and freezing processes of a water droplet on different cold cylindrical surfaces. Int J Heat Mass Transf 113:318-323. https://doi.org/10.1016/j.jijheatmasstransfer.2017.05.111

7. Wang Y, Wang Z-G (2018) Sessile droplet freezing on polished and micro-micro-hierarchical silicon surfaces. Appl Thermal Eng 137:66-73. https://doi.org/10.1016/j.applthermaleng.2018.03.068

8. Pan Y, Shi K, Duan X, Naterer GF (2019) Experimental investigation of water droplet impact and freezing on micropatterned stainless steel surfaces with varying wettabilities. Int J Heat Mass Transf 129:953-964. https://doi.org/10.1 016/j.jheatmasstransfer.2018.10.032

9. Yamada Y, Onishi G, Horibe A (2019) Sessile droplet freezing on hydrophobic structured surfaces under cold ambient conditions. Langmuir 35(50):16401-16406. https://doi.org/10.1021/acs.langmuir.9b01173

10. Ding B, Wang H, Zhu X, Chen R, Liao Q (2019) Water droplet impact on superhydrophobic surfaces with various inclinations and supercooling degrees. Int J Heat Mass Transf 138:844-851. https://doi.org/10.1016/j.jiheatmasstransfer.2 019.04 .106

11. Ju J, Yang Z, Yi X, Jin Z (2019) Experimental investigation of the impact and freezing processes of a hot water droplet on an ice surface. Phys Fluids 31(5):057107. https://doi.org/10.1063/1.5094691

12. Hindmarsh JP, Russell AB, Chen XD (2003) Experimental and numerical analysis of the temperature transition of a suspended freezing water droplet. Int J Heat Mass Transf 46(7):1199-1213. https://doi.org/10.1016/s0017-9310(02)00399$x$

13. Chaudhary G, Li R (2014) Freezing of water droplets on solid surfaces: an experimental and numerical study. Exp Thermal Fluid Sci 57:86-93. https://doi.org/10.1016/j.expthermflusci.2014.04.007

14. Zhang X, Liu X, Min J, Wu X (2019) Shape variation and unique tip formation of a sessile water droplet during freezing Appl Therm Eng 147:927-934. https://doi.org/10.1016/j.applthermaleng.2018.09.040

15. Zhang X, Liu X, Wu X, Min J (2020) Impacting-freezing dynamics of a supercooled water droplet on a cold surface: rebound and adhesion. Int J Heat Mass Transf 158:119997. https://doi.org/10.1016/j.ijheatmasstransfer.2020.119997

16. Bodaghkhani A, Duan X (2020) Water droplet freezing on cold surfaces with distinct wetabilities. Heat Mass Transf 57(5): 1-10. https://doi.org/10.1007/s00231-020-02984-w

17. Jr P (2002) Nucleation, growth and surface movement of a condensing sessile droplet. Colloids Surf A Physicochem Eng Asp 206(1-3):157-165

18. Yamanaka S, Ito N, Akiyama K, Shimosaka A, Shirakawa Y, Hidaka J (2012) Heterogeneous nucleation and growth mechanism on hydrophilic and hydrophobic surface. Adv Powder Technol 23(2):268-272. https://doi.org/10.1016/j.apt.2 012.01 .002

19. Kožíšek Z (2019) Crystallization in small droplets: competition between homogeneous and heterogeneous nucleation. J Cryst Growth 522:53-60. https://doi.org/10.1016/j.jcrysgro.2019.06.007 
20. Bostanov V, Mladenova E, Kashchiev D (2000) Nucleation rate in electrocrystallization of cadmium on the $\mathrm{Cd}(0001)$ crystal face. J Electroanal Chem 481(1):7-12. https://doi.org/10.1016/S0022-0728(99)00471-4

21. Meng Z, Zhang P (2020) Dynamic propagation of ice-water phase front in a supercooled water droplet. Int J Heat Mass Transfer 152:119468

22. Murray BJ, Wilson TW, Broadley SL, Wills RH (2010) Heterogeneous freezing of water droplets containing kaolinite and montmorillonite particles. Atmospheric Chem Phys Discussions 10(4):9695-9729

23. Wang Y, Chen S (2015) Droplets impact on textured surfaces: mesoscopic simulation of spreading dynamics. Appl Surf Sci 327:159-167. https://doi.org/10.1016/j.apsusc.2014.11.148

24. Zhao J, Chen S, Liu Y (2016) Droplets motion on chemically/topographically heterogeneous surfaces. Mol Simul 42(17): 1452-1459. https://doi.org/10.1080/08927022.2016.1198478

25. Wang L, Zhang R, Zhang X, Hao P (2017) Numerical simulation of droplet impact on textured surfaces in a hybrid state. Microfluidics Nanofluidics 21(4):61. https://doi.org/10.1007/s10404-017-1900-0

26. Lin C, Chen S, Xiao L, Liu Y (2018) Tuning drop motion by chemical chessboard-patterned surfaces: a many-body dissipative particle dynamics study. Langmuir 34(8):2708-2715. https://doi.org/10.1021/acs.langmuir.7b04162

27. Wang L, Dai J, Hao P, He F, Zhang X (2020) Mesoscopic dynamical model of ice crystal nucleation leading to droplet freezing. ACS omega 5(7):3322-3332. https://doi.org/10.1021/acsomega.9b03415

28. Wang $\mathrm{C}$, Wu X, Zhang H, Hao P, He F, Zhang X (2021) A many-body dissipative particle dynamics study of eccentric droplets impacting inclined fiber. Phys Fluids 33(4):042001. https://doi.org/10.1063/5.0044065

29. Hoogerbrugge PJ, Koelman J (1992) Simulating microscopic hydrodynamic phenomena with dissipative particle dynamics. Europhys Lett 19(3):155-160. https://doi.org/10.1209/0295-5075/19/3/001

30. Groot RD, Warren PB (1997) Dissipative particle dynamics: bridging the gap between atomistic and mesoscopic simulation. J Chem Phys 107(11):4423-4435. https://doi.org/10.1063/1.474784

31. Zhang X, Wu X, Min J, Liu X (2017) Modelling of sessile water droplet shape evolution during freezing with consideration of supercooling effect. Appl Therm Eng 125:644-651. https://doi.org/10.1016/j.applthermaleng.2017.07.017

32. Liu Y, Li X, Jin J, Liu J, Yan Y, Han Z, Ren L (2017) Anti-icing property of bio-inspired micro-structure superhydrophobic surfaces and heat transfer model. Appl Surf Sci 400:498-505. https://doi.org/10.1016/j.apsusc.2016.12.219

\section{Publisher's Note}

Springer Nature remains neutral with regard to jurisdictional claims in published maps and institutional affiliations.

\section{Submit your manuscript to a SpringerOpen ${ }^{\circ}$ journal and benefit from:}

- Convenient online submission

- Rigorous peer review

- Open access: articles freely available online

- High visibility within the field

- Retaining the copyright to your article

Submit your next manuscript at $\boldsymbol{\nabla}$ springeropen.com 